Article

\title{
Synthesis and Cytotoxicity Testing of New Amido-Substituted Triazolopyrrolo[2,1-c][1,4]benzodiazepine (PBDT) Derivatives
}

\section{Kumaraswamy Sorra ${ }^{1,2, \dagger}$, Chi-Fen Chang ${ }^{3, \dagger}$, Srinivas Pusuluri ${ }^{1}$, Khagga Mukkanti ${ }^{2}$, Min-Chiau Laiu ${ }^{4}$, Bo-Ying Bao ${ }^{5}$, Chia-Hao Su ${ }^{4, *}$ and Ta-Hsien Chuang ${ }^{5, *}$}

1 Medicinal Chemistry Laboratory, GVK Biosciences Private Limited, Plot No. 5C, IDA Uppal, Hyderabad 500039, AP, India; E-Mail: pusulurisrinivas@gvkbio.com (S.P.)

Chemistry Division, Institute of Science and Technology, JNT University, Kukatpally, Hyderabad 500072, AP, India

3 Department of Anatomy and Cell Biology, College of Medicine, National Taiwan University, 1-1, Jen-Ai Road, Taipei 100, Taiwan; E-Mail: changchifen@ntu.edu.tw

4 Center for Translational Research in Biomedical Sciences, Kaohsiung Chang Gung Memorial Hospital, Kaohsiung 833, Taiwan; E-Mail: a0910758792@hotmail.com

5 School of Pharmacy, China Medical University, Taichung 40402, Taiwan; E-Mail: bao@mail.cmu.edu.tw

$\dagger$ These authors contributed equally to this work.

* Authors to whom correspondence should be addressed; E-Mails: chiralsu@gmail.com (C.-H.S.); thchuang@mail.cmu.edu.tw (T.-H.C.); Tel.: +886-7-731-7123 (ext. 8592) (C.-H.S.); Fax: +886-7-731-7123 (ext. 8569) (C.-H.S.); Tel.: +886-4-2205-3366 (ext. 5150) (T.-H.C.); Fax: +886-4-2203-1075 (T.-H.C.).

Received: 1 June 2012; in revised form: 16 July 2012 / Accepted: 18 July 2012 /

Published: 25 July 2012

\begin{abstract}
A series of amido-substituted triazolopyrrolo[2,1-c][1,4]benzodiazepine (PBDT) derivatives was synthesized from isatoic anhydride, and their cytotoxicity against the MRC-5 and Mahlavu cell lines was evaluated. The results suggest that compound PBDT-7i with the meta-trifluoromethylbenzoyl substituent can selectively inhibit the growth of Mahlavu cells and has low toxicity towards MRC-5 cells.
\end{abstract}

Keywords: triazolopyrrolobenzodiazepine; Lawesson's reagent; cytotoxicity; Mahlavu celles; MRC-5 cells 


\section{Introduction}

Cancer is a leading cause of death globally, accounting for 7.6 million deaths in 2008. Hepatocellular carcinoma (HCC) is one of the most common cancers worldwide and occurs at a high rate (3-5\% annually) in patients with chronic viral hepatitis and cirrhosis. HCC is also a major cause of morbidity and mortality in patients with advanced liver disease [1,2]. Therefore, there is a desire to design and develop more potent therapeutic agents for cancer. We are interested in investigating $N$-bridged heterocycles composed of two bioactive heterocyclic moieties, such as benzodiazepines and triazoles, in a single molecular scaffold because such bridged molecules are endowed with a variety of biological activities and have a wide range of therapeutic properties.

Among pharmacologically important heterocyclic compounds, benzodiazepines are useful scaffolds that are widely applied for various therapeutic purposes [3-8]. Pyrrolo[2,1-c][1,4]benzodiazepines (PBDs) are found in natural antitumor antibiotics isolated from Streptomyces species; these natural antitumor antibiotics include anthramycin (1), tomaymycin, chicamycin A, DC-81 (2), mazethramycin, abbeymycin (3), neothramycin A and B and porothramycin B (Figure 1) [9]. PBDs can recognize and bind to specific DNA sequences and are potential regulators of gene expression. These compounds have possible applications as therapeutic agents for the treatment of genetic disorders including cancer [10]. Benzodiazepines made up of fused tri- and tetraheterocyclic rings have attracted attention in the area of medicinal chemistry; midazolam (4), flumazenil and estazolam (5) have attracted attention in the area of CNS disorders, and bretazenil (6) has emerged for the treatment of neurodegenerative diseases [11-15] (Figure 1).

Figure 1. Members of the benzodiazepine family.<smiles>Cc1ccc2c(c1O)N[C@H](O)[C@H]1CC(/C=C/C(N)=O)=CN1C2=O</smiles>

Anthramycin, 1<smiles>Cc1ncc2n1-c1ccc(Cl)cc1C(c1ccccc1F)=NC2</smiles>

Midazolam, 4

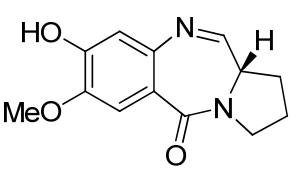

DC-81, 2<smiles>COC1Nc2ccccc2C(=O)N2C[C@H](O)C[C@]12C</smiles>

Abbeymycin, 3

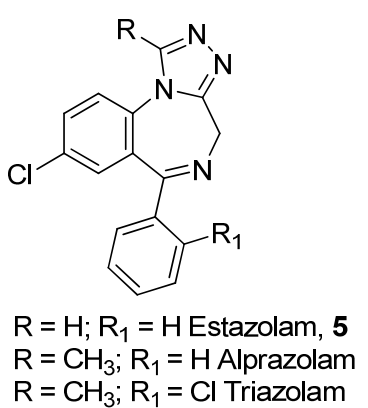<smiles>CC(C)(C)OC(=O)c1ncn2c1[C@]1(C)CCCN1C(=O)c1c(Br)cccc1-2</smiles>

Bretazenil, 6

In addition, 1,2,4-triazole and its derivatives are being used in a variety of therapeutic applications due to their antibacterial, antifungal, anticancer, antitumor, anticonvulsant, anti-inflammatory and analgesic properties [16,17]. 1,2,4-Triazoles fused with other heterocyclic moieties possess a broad spectrum of biological activities [18].

Owing to the biological significance of these two classes of compounds, we planned to synthesize a combined molecular framework. We report herein the synthesis of tetracyclic triazolopyrrolo[2,1-c] 
$[1,4]$ benzodiazepines (PBDTs) and their amido-substituted derivatives. The cytotoxicity of the newly synthesized derivatives was investigated using the Mahlavu (human hepatocellular carcinoma) and MRC-5 (normal human fibroblast) cell lines.

\section{Results and Discussion}

\subsection{Synthesis}

The synthesis strategy for constructing the tetracyclic 1-amido-substituted triazolopyrrolo[2,1-c] $[1,4]$ benzodiazepin-8-one derivates PBDT-7a-k is shown in Scheme 1. The key intermediate 11 was prepared in four steps. First, pyrrolo-benzodiazepinedione $\mathbf{8}$ was obtained by the cyclocondensation of L-proline with isatoic anhydride. The corresponding mono-thiolactam 9 was produced by thiation employing Lawesson's reagent in toluene at $70{ }^{\circ} \mathrm{C}$ [19]. Subsequently, the thiolactam 9 was converted into 11-hydrazinopyrrolo[2,1-c][1,4]benzodiazepine (10) using hydrazine hydrate in ethanol at $\mathrm{rt}$, and cyclization with cyanogen bromide afforded the triazolo PBD 11 [20,21]. Then, a series of amido-substituted triazolo-fused PBDs (compounds PBDT-7a-k) was synthesized from the tetracyclic intermediate $\mathbf{1 1}$ by coupling these molecules with the corresponding carboxylic acids using HATU as a coupling agent in the presence of Hünig's base (Table 1).

Scheme 1. Synthesis of triazolo-fused PBD and derivatives.<smiles>O=c1[nH]c2ccccc2c(=O)o1</smiles><smiles>C[13CH][13CH3]</smiles><smiles>O=C1c2ccccc2NC(=O)N2CCC[C@H]12</smiles><smiles>CPC</smiles><smiles>O=C1c2ccccc2NC(=S)[C@H]2CCCN12</smiles><smiles>[R]C(=O)Nc1nnc2n1-c1ccccc1C(=O)N1CCC[C@@H]1[C@H]2P</smiles><smiles>[R20]C(=O)O</smiles><smiles>Nc1nnc2n1-c1ccccc1C(=O)N1CCC[C@H]21</smiles><smiles>C1CCCCC1</smiles><smiles>NNC1=Nc2ccccc2C(=O)N2CCC[C@H]12</smiles>

$$
\begin{aligned}
& 7 \mathbf{a}=\mathrm{R}=-\mathrm{CMe}_{3} ; \quad \mathbf{7 g}=\mathrm{R}=m-\mathrm{CN}-\mathrm{Ph} \\
& \mathbf{7 b}=\mathrm{R}=\mathrm{Ph} ; \quad \mathbf{h}=\mathrm{R}=m-\mathrm{Cl}-\mathrm{Ph} \\
& \mathbf{7 c}=\mathrm{R}=o-\mathrm{F}-\mathrm{Ph} ; \quad 7 \mathbf{i}=\mathrm{R}=m-\mathrm{CF}_{3}-\mathrm{Ph} \\
& \mathbf{7 d}=\mathrm{R}=m-\mathrm{F}-\mathrm{Ph} ; \mathbf{7}=\mathrm{R}=3^{\prime}-\text { pyridinyl } \\
& \mathbf{7 e}=\mathrm{R}=p-\mathrm{F}-\mathrm{Ph} ; \quad \mathbf{7 k}=\mathrm{R}=2^{\prime}-\text { thiophene } \\
& \mathbf{7 f}=\mathrm{R}=m-\mathrm{OMe}-\mathrm{Ph}
\end{aligned}
$$

Reagents and conditions: (a) L-Proline, DMF, $140{ }^{\circ} \mathrm{C}, 5 \mathrm{~h}, 85 \%$ yield; (b) Lawesson's reagent, toluene, $70{ }^{\circ} \mathrm{C}, 5 \mathrm{~h}, 86 \%$ yield; (c) $98 \% \mathrm{~N}_{2} \mathrm{H}_{4} \cdot \mathrm{H}_{2} \mathrm{O}$, EtOH, rt, 4 h, $70 \%$ yield; (d) BrCN, EtOH, rt, 12 h, 81\% yield; (e) (i) PBDT-7a: pivaloyl chloride, pyridine, THF, $0{ }^{\circ} \mathrm{C}, 45 \mathrm{~min}$; (ii) PBDT-7b-k: HATU, $i$ - $\mathrm{Pr}_{2} \mathrm{NEt}$, DMF, $50{ }^{\circ} \mathrm{C}, 15-45 \mathrm{~min}$. 
Table 1. Series of triazolo-pyrrolo-benzodiazepine derivatives PBDT-7a-k synthesized from compound $\mathbf{1 1}$ by coupling with the corresponding carboxylic acids.

\begin{tabular}{clccc}
\hline S.No. & \multicolumn{1}{c}{ Acid (12a-k) } & PBDT-7 & Time (min) & Yield (\%) \\
\hline 1 & $\mathrm{R}=t$-butyl $*$ & $\mathbf{7 a}$ & 45 & 65 \\
2 & $\mathrm{R}=$ phenyl & $\mathbf{7 b}$ & 20 & 80 \\
3 & $\mathrm{R}=o$-fluorophenyl & $\mathbf{7 c}$ & 30 & 75 \\
4 & $\mathrm{R}=m$-fluorophenyl & $\mathbf{7 d}$ & 30 & 80 \\
5 & $\mathrm{R}=p$-fluorophenyl & $\mathbf{7 e}$ & 30 & 75 \\
6 & $\mathrm{R}=m$-methoxyphenyl & $\mathbf{7 f}$ & 15 & 70 \\
7 & $\mathrm{R}=m$-cyanophenyl & $\mathbf{7 g}$ & 40 & 65 \\
8 & $\mathrm{R}=m$-chlorophenyl & $\mathbf{7 h}$ & 30 & 65 \\
9 & $\mathrm{R}=m$-trifluoromethylphenyl & $\mathbf{7 i}$ & 15 & 70 \\
10 & $\mathrm{R}=3^{\prime}$-pyridinyl & $\mathbf{7 j}$ & 30 & 75 \\
11 & $\mathrm{R}=2^{\prime}$-thiophene & $\mathbf{7 k}$ & 30 & 72 \\
\hline
\end{tabular}

* Pivaloyl chloride was used.

\subsection{Cytotoxicity}

The cytotoxic activities of the newly synthesized amido-substituted triazolopyrrolo [2,1-c][1,4] benzodiazepines PBDT-7a-k were tested against Mahlavu and MRC-5 cells. After incubation of the cells with the compounds at concentrations of 3.12, 6.25, 12.50, 25.0, 50.0 and $100 \mu \mathrm{M}$ for $48 \mathrm{~h}$, the cell growth and viability were assessed by using CCK- 8 assay. The results are shown in Figures 2 and 3 . Our initial results showed that percentages of viable Mahlavu and MRC-5 cells exposed to the compounds PBDT-7a,b and PBDT-7j, $\mathbf{k}$ were above 75\%, although the concentration was increased to $100 \mu \mathrm{M}$. This revealed that the aliphatic and aromatic amides seem no significant difference observed in cytotoxicity between Mahlavu and MRC-5 cell lines. Intriguingly, cells exposed to compounds with various substituents [-F (PBDT-7c-e), -OMe (PBDT-7f), -CN (PBDT-7g), -Cl (PBDT-7h) and -CF 3 (PBDT-7i)] on the phenyl ring of the PBDT exhibited different viabilities for both cell lines. Compounds with the fluoro substituent at various positions (compounds PBDT-7c-e) exhibited different levels of toxicity against Mahlavu and MRC-5 cells. The viability of Mahlavu cells exposed to the compound with a fluoro substituent at the meta-position (compound PBDT-7d) was approximately $61 \%$ at $100 \mu \mathrm{M}$, whereas the ortho- and para-fluoro-substituted compounds PBDT-7c and e had little effect at the same concentration.

Based on the preliminary results, we selected to further investigate the meta-position of the phenyl group (compounds PBDT-7f-i). The cell viabilities were reduced in the presence of the chloro-substituted PBDT-7h in a dose-dependent manner, and the survival rate of Mahlavu cells was below 50\% at $50 \mu \mathrm{M}$. PBDT-7h was a potent inhibitor of Mahlavu cells, but was also highly toxic for normal cells. To increase the specificity, we evaluated other three meta-substituted PBDTs, including compounds with the -OMe (PBDT-7f), -CN (PBDT-7g) and - $\mathrm{CF}_{3}$ (PBDT-7i) substituents. It should be noted that the cell viabilities of Mahlavu cells were decreased to $37 \%, 36 \%$, and $2 \%$ at $100 \mu \mathrm{M}$, respectively, and the $\mathrm{IC}_{50}$ values of PBDT-7g and PBDT-7i were $50 \mu \mathrm{M}$ for Mahlavu cells. These compounds were slightly toxic to MRC-5 cells, with cell viabilities above $70 \%$ at $50 \mu \mathrm{M}$. These revealed compound PBDT-7i with the meta-trifluoromethylbenzoyl substituent can selectively inhibit 
the growth of Mahlavu cells and is low-toxic to MRC-5 cells. This novel compound certainly deserves further careful investigation to determine its mechanism of action.

Figure 2. PBDT-7a-k induced the death of Mahlavu cells. The concentration-dependent effects of PBDT-7a-k on human hepatocellular carcinoma cells. Mahlavu cells were treated with PBDT-7 series compounds for $48 \mathrm{~h}$, and survival was assessed using a CCK-8 assay (mean \pm SEM, $\mathrm{n}=6$ ).

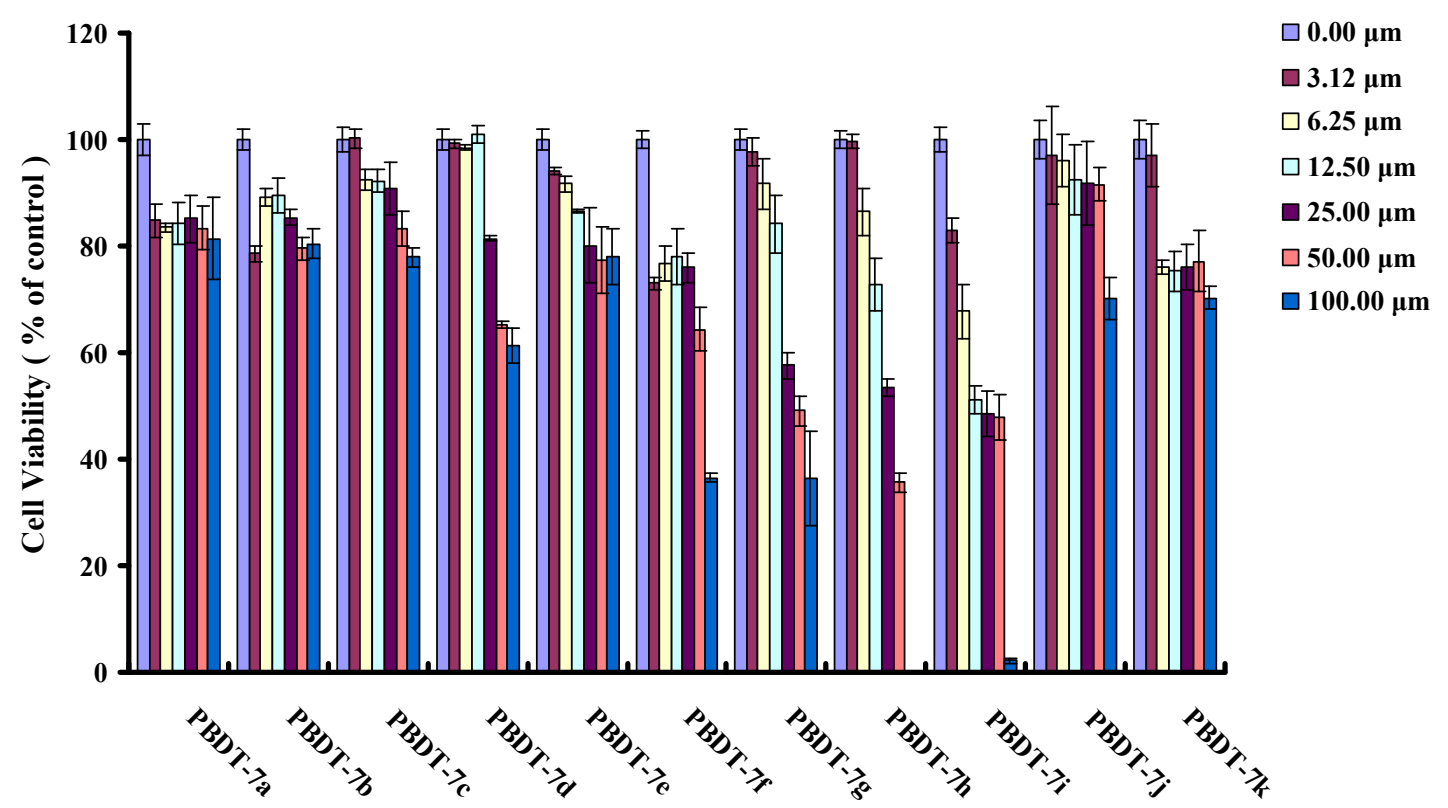

Figure 3. The cytotoxicity assay of PBDT-7a-k against MRC-5 cells. The concentration-dependent effect of PBDT-7a-k on normal human fibroblast cells. MRC-5 cells were treated with PBDT-7 series compounds for $48 \mathrm{~h}$, and survival was assessed using a CCK-8 assay (mean \pm SEM, $\mathrm{n}=6$ ).

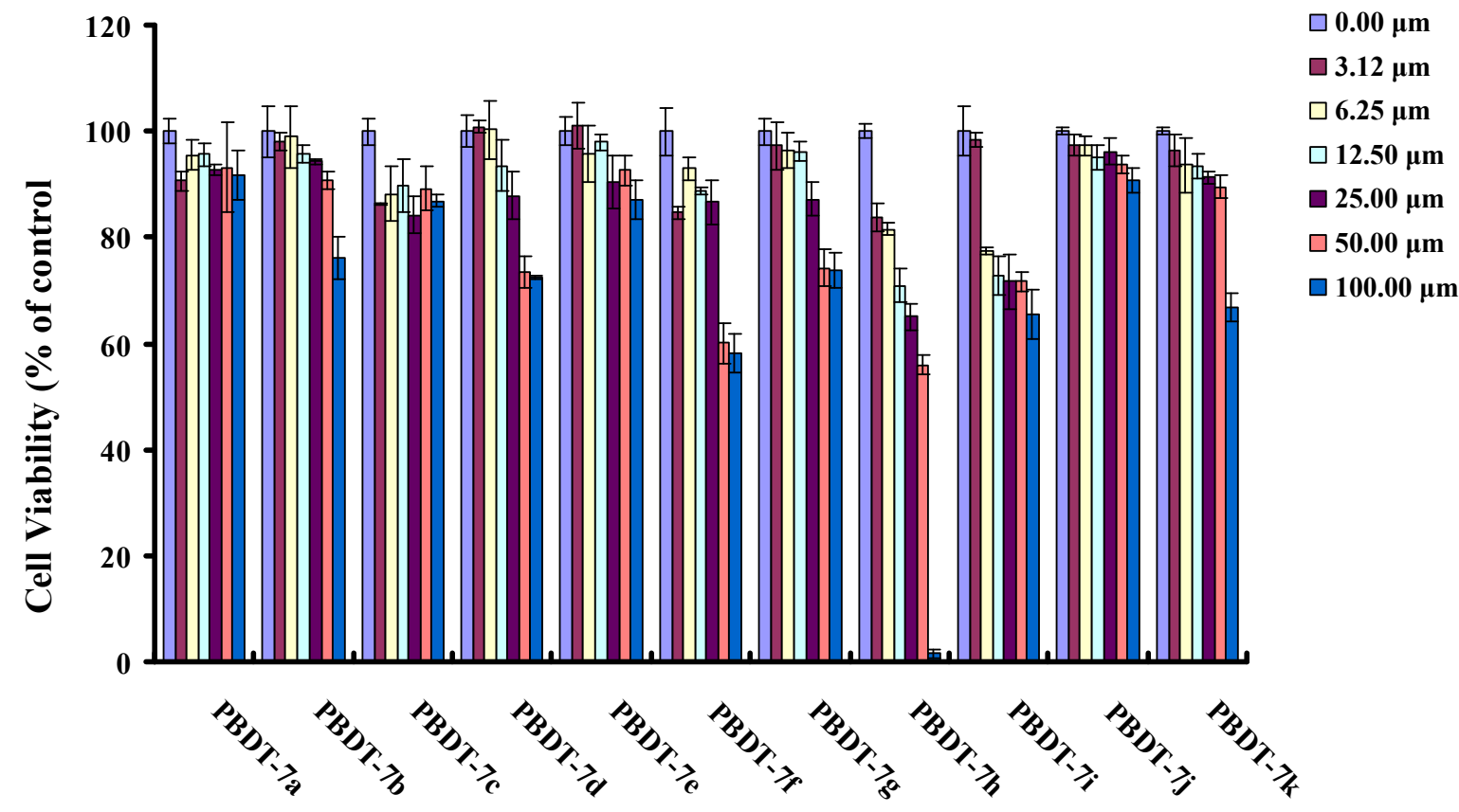




\section{Experimental}

\subsection{General}

Melting point (mp) determinations were performed by using a Mel-temp apparatus and are uncorrected. ${ }^{1} \mathrm{H}$ - and ${ }^{13} \mathrm{C}$-NMR spectra were recorded in DMSO- $d_{6}$ (unless otherwise specified) on a Varian Unity instrument at room temperature at $400 / 100 \mathrm{MHz}$, respectively. Chemical shifts are reported in $\delta$ parts per million (ppm) downfield from tetramethylsilane (TMS) with reference to internal solvent and coupling constants are expressed in Hz. Mass spectra were obtained on a Micromass QuattroMicro ${ }^{\mathrm{TM}} \mathrm{API}$-autospectrometer using the APCI technique. HRMS TOF-ES mass spectra were recorded on a Waters-Alliance 2695 Separation Module/Q-TOF Micromass. Infrared (IR) spectra (KBr disks) were recorded on a Perkin Elmer FT-IR spectrometer. Optical rotations $\left([\alpha]_{\mathrm{D}}\right)$ were measured on a JASCO P-1030 polarimeter. Liquid chromatography-mass spectrometry (LCMS) data was generated on a Waters acquity UPLC photodiode array detector (PDA) system using the ESI technique.

\subsection{Synthesis}

(11aS)-2,3-Dihydro-1H-pyrrolo[2,1-c][1,4]benzodiazepine-5,11(10H,11aH)-dione (8). A suspension of isatoic anhydride $(10.0 \mathrm{~g}, 61.34 \mathrm{mmol})$ and L-proline $(7.06 \mathrm{~g}, 61.34 \mathrm{mmol})$ in $N, N$-dimethyl formamide $(50 \mathrm{~mL})$ was heated to $140{ }^{\circ} \mathrm{C}$ for $5 \mathrm{~h}$. The solvent was removed in vacuo and the residue was taken up in water. The precipitate was collected and dried to give 8 (11.30 g, 85\% yield) as an off-white solid. mp $214-216{ }^{\circ} \mathrm{C}$; $[\alpha]_{\mathrm{D}}^{23}+497^{\circ}$ (c 1.03, MeOH); IR $v_{\max } 3200,1700,1630 \mathrm{~cm}^{-1}$; ${ }^{1} \mathrm{H}-\mathrm{NMR}\left(\mathrm{CDCl}_{3}\right): \delta$ 1.97-2.08 $(3 \mathrm{H}, \mathrm{m}), 2.72-2.80(1 \mathrm{H}, \mathrm{m}), 3.56-3.63(1 \mathrm{H}, \mathrm{m}), 3.77-3.82(1 \mathrm{H}, \mathrm{m})$, $4.07(1 \mathrm{H}, \mathrm{d}, J=6.2 \mathrm{~Hz}), 7.04(1 \mathrm{H}, \mathrm{d}, J=8.0 \mathrm{~Hz}), 7.23-7.27(1 \mathrm{H}, \mathrm{m}), 7.45-7.47(1 \mathrm{H}, \mathrm{m}), 7.99(1 \mathrm{H}, \mathrm{dd}$, $J=8.0,1.6 \mathrm{~Hz}), 8.92(1 \mathrm{H}$, br s, $\mathrm{NH}) ;{ }^{13} \mathrm{C}-\mathrm{NMR}\left(\mathrm{CDCl}_{3}\right): \delta 23.6,26.4,47.4,56.8,121.2,125.2,127.3$, 131.3, 132.6, 135.5, 165.6, 171.5; LCMS $\left(\mathrm{ES}^{+}\right): m / z 217$ [M+H] $]^{+}$; HRMS (TOF ES ${ }^{+}$): $\mathrm{MH}^{+}$, calcd for $\mathrm{C}_{12} \mathrm{H}_{13} \mathrm{~N}_{2} \mathrm{O}_{2}: 217.0971$; found: $217.0972[\mathrm{M}+\mathrm{H}]^{+}$.

(11aS)-11-Thioxo-1,2,3,10,11,11a-hexahydro-5H pyrrolo [2,1-c][1,4]ben-zodiazepin-5-one (9). To a suspension of dilactam $8(5.0 \mathrm{~g}, 23.10 \mathrm{mmol})$ in dry toluene $(300 \mathrm{~mL})$ was added Lawesson's reagent ( $4.7 \mathrm{~g}, 11.55 \mathrm{mmol})$. The yellow suspension was heated to $70{ }^{\circ} \mathrm{C}$ for $5 \mathrm{~h}$. After this time a yellow solid has precipitated which was recrystallized from ethanol to yield pure 9 ( $4.6 \mathrm{~g}, 86 \%$ yield) as yellow crystals. mp $291-292{ }^{\circ} \mathrm{C} ;[\alpha]_{\mathrm{D}}{ }^{27}+729.1^{\circ}$ (c 1.08, DMSO); IR $v_{\max } 3446,1616,1605,1521,886 \mathrm{~cm}^{-1}$; ${ }^{1} \mathrm{H}-\mathrm{NMR}\left(\mathrm{CDCl}_{3}\right): \delta$ 1.97-2.03 $(1 \mathrm{H}, \mathrm{m}), 2.08-2.18(1 \mathrm{H}, \mathrm{m}), 2.22-2.31(1 \mathrm{H}, \mathrm{m}), 3.08-3.13(1 \mathrm{H}, \mathrm{m})$, 3.57-3.64 (1H, m), 3.77-3.80 (1H, m), $4.22(1 \mathrm{H}, \mathrm{d}, J=8.0 \mathrm{~Hz}), 7.05(1 \mathrm{H}, \mathrm{d}, J=8.0 \mathrm{~Hz}), 7.33-7.37$ $(1 \mathrm{H}, \mathrm{m}), 7.49-7.53(1 \mathrm{H}, \mathrm{m}), 8.04(1 \mathrm{H}, \mathrm{d}, J=8.0 \mathrm{~Hz}), 9.73\left(1 \mathrm{H}\right.$, br s, NH); ${ }^{13} \mathrm{C}-\mathrm{NMR}: \delta 22.6,28.9$, 46.8, 59.7, 121.8, 125.6, 127.7, 130.2, 132.1, 136.4, 164.1, 201.9; LCMS $\left(\mathrm{ES}^{+}\right) \mathrm{m} / z 233\left(\mathrm{M}+\mathrm{H}^{+}\right)$; HRMS (TOF ES ${ }^{+}$): $\mathrm{MH}^{+}$, calcd for $\mathrm{C}_{12} \mathrm{H}_{13} \mathrm{~N}_{2} \mathrm{OS}$ : 233.0749; found 233.0738 [M+H] $]^{+}$.

(S)-11-Hydrazinyl-2,3-dihydro-1H-benzo[e]pyrrolo[1,2-a][1,4]diazepin-5(11aH)-one (10). A solution of compound $9(2.5 \mathrm{~g}, 10.77 \mathrm{mmol})$ and $98 \%$ hydrazine monohydrate $(0.65 \mathrm{~g}, 12.98 \mathrm{mmol})$ in ethanol $(25 \mathrm{~mL})$ was stirred for $4 \mathrm{~h}$ at room temperature. The solvent was removed under reduced pressure and the residue was taken up in water. The precipitate was collected, dried and washed with diethyl ether to 
yield compound 10 (1.74 g, 70\% yield) as a white solid. mp $178-180{ }^{\circ} \mathrm{C}$; IR $v_{\max } 3370,3330,1640$, $1600 \mathrm{~cm}^{-1} ;[\alpha]_{\mathrm{D}}{ }^{26}+154^{\circ}\left(c\right.$ 1.02, MeOH). ${ }^{1} \mathrm{H}-\mathrm{NMR}: \delta 1.78-1.82(2 \mathrm{H}, \mathrm{m}), 2.14-2.18(1 \mathrm{H}, \mathrm{m})$, 2.46-2.50 (1H, m), $3.02(1 \mathrm{H}, \mathrm{d}, J=11.2 \mathrm{~Hz}), 3.34(1 \mathrm{H}, \mathrm{d}, J=11.2 \mathrm{~Hz}), 4.62-4.66(1 \mathrm{H}, \mathrm{m}), 6.32-6.37$ $(1 \mathrm{H}$, br s, NH), $7.15(1 \mathrm{H}, \mathrm{t}, J=7.8 \mathrm{~Hz}) ; 7.31(1 \mathrm{H}, \mathrm{d}, J=7.8 \mathrm{~Hz}) ; 7.47(1 \mathrm{H}, \mathrm{t}, J=7.8 \mathrm{~Hz}) ; 7.80(1 \mathrm{H}, \mathrm{d}$, $J=7.8 \mathrm{~Hz}) ;{ }^{13} \mathrm{C}-\mathrm{NMR}: \delta 23.6,26.9,46.8,51.0,122.8,126.0,127.0,129.0,132.0,134.2,151.0,162.0$; LCMS (ES $\left.{ }^{+}\right): m / z 231[\mathrm{M}+\mathrm{H}]^{+}$; HRMS (TOF ES ${ }^{+}$): $\mathrm{MH}^{+}$, calcd for $\mathrm{C}_{12} \mathrm{H}_{15} \mathrm{~N}_{4} \mathrm{O}: 231.1205$; found $231.1207[\mathrm{M}+\mathrm{H}]^{+}$.

(13aS)-3-Amino-11,12,13,13a-tetrahydro-9H-benzo[e]pyrrolo[1,2-a][1,2,4]triazolo[3,4-c][1,4]diazepin-9one (11). A solution of compound $10(1.0 \mathrm{~g}, 4.35 \mathrm{mmol})$ and cyanogen bromide $(0.47 \mathrm{~g}, 4.78 \mathrm{mmol})$ in ethanol $(10 \mathrm{~mL})$ was stirred for $12 \mathrm{~h}$ at room temperature. The reaction mixture was neutralized with $10 \%$ aqueous potassium bicarbonate, the precipitated solid was filtered and recrystallised from EtOH to give pure $11(0.90 \mathrm{~g}, 81 \%$ yield $)$ as white crystals. mp $210-212{ }^{\circ} \mathrm{C} ;[\alpha]_{\mathrm{D}}{ }^{26}+104(c 1.02, \mathrm{MeOH})$; IR $v_{\max }$ 3339, 1624, 1467, $763 \mathrm{~cm}^{-1}$; ${ }^{1} \mathrm{H}-\mathrm{NMR}: \delta$ 1.94-1.99 $(2 \mathrm{H}, \mathrm{m}), 2.21-2.31(1 \mathrm{H}, \mathrm{m}), 2.77-2.80$ $(1 \mathrm{H}, \mathrm{m}), 3.50-3.64(2 \mathrm{H}, \mathrm{m}), 4.60(1 \mathrm{H}, \mathrm{dd}, J=8.0,3.2 \mathrm{~Hz}), 6.09(1 \mathrm{H}$, br s, NH), 7.49-7.53 (1H, m), 7.68-7.70 (1H, m), $7.86(1 \mathrm{H}, \mathrm{d}, J=8.0 \mathrm{~Hz}) ;{ }^{13} \mathrm{C}-\mathrm{NMR}: \delta 23.2,26.2,46.9,51.1,122.7,127.4,129.6$, 130.6, 131.1, 132.1, 150.5, 154.6, 163.7; LCMS $\left(\mathrm{ES}^{+}\right): \mathrm{m} / z 256[\mathrm{M}+\mathrm{H}]^{+}$; HRMS (TOF ES ${ }^{+}$): $\mathrm{MH}^{+}$, calcd for $\mathrm{C}_{13} \mathrm{H}_{14} \mathrm{~N}_{5} \mathrm{O}$ : 256.1139; found 256.1135 [M+H] $]^{+}$.

((13aS)-9-Oxo-11,12,13,13a-tetrahydro-9H-benzo[e]pyrrolo[1,2-a][1,2,4]triazolo[3,4-c][1,4]diazepin3-yl)pivalamide (7a). To a solution of compound $11(0.1 \mathrm{~g}, 0.39 \mathrm{mmol})$ and pyridine $(0.2 \mathrm{~mL}, 2.48 \mathrm{mmol})$ in $\mathrm{T}(2 \mathrm{~mL})$ was added pivaloyl chloride $(0.058 \mathrm{~mL}, 47.05 \mathrm{mmol})$ dropwise at $0{ }^{\circ} \mathrm{C}$. The mixture was stirred at $0{ }^{\circ} \mathrm{C}$ for $45 \mathrm{~min}$ and filtered. The filtrate was concentrated in vacuo, the residue was dissolved in ethyl acetate, washed sequentially with water and saturated sodium bicarbonate solution, dried over sodium sulfate and concentrated under reduced pressure to give compound $7 \mathbf{a}(0.086 \mathrm{~g}$, $65 \%$ yiled) as an off-white solid. mp $213-215{ }^{\circ} \mathrm{C}$; IR $v_{\max } 3296,2957,1687,1630 \mathrm{~cm}^{-1} ;{ }^{1} \mathrm{H}-\mathrm{NMR}: \delta$ $1.11(9 \mathrm{H}, \mathrm{s}), 2.01-2.04(2 \mathrm{H}, \mathrm{m}), 2.31-2.39(1 \mathrm{H}, \mathrm{m}), 2.81-2.89(1 \mathrm{H}, \mathrm{m}), 3.50-3.65(2 \mathrm{H}, \mathrm{m}), 4.79-4.83$ $(1 \mathrm{H}, \mathrm{m}), 7.42(1 \mathrm{H}, \mathrm{d}, J=8.0 \mathrm{~Hz}), 7.55(1 \mathrm{H}, \mathrm{d}, J=8.0 \mathrm{~Hz}), 7.68(1 \mathrm{H}, \mathrm{d}, J=6.8 \mathrm{~Hz}), 7.89(1 \mathrm{H}, \mathrm{d}$, $J=6.8 \mathrm{~Hz}), 10.26\left(1 \mathrm{H}\right.$, br s, NH); LCMS $\left(\mathrm{ES}^{+}\right): m / z 340[\mathrm{M}+\mathrm{H}]^{+}$.

\subsection{General Procedure for Amide Coupling: Preparation of $\mathbf{7 b}-\mathbf{k}$}

To a stirred solution of compound $11(0.1 \mathrm{~g}, 0.39 \mathrm{mmol})$ and $N, N$-diisopropylethylamine $(0.17 \mathrm{~mL}$, $0.98 \mathrm{mmol})$ in $N, N$-dimethylformamide $(2 \mathrm{~mL})$ was added the appropriate carboxylic acid $\mathbf{1 2 b}-\mathbf{k}$ (43.14 mmol) and 2-(7-aza-1H-benzotriazole-1-yl)-1,1,3,3-tetramethyluronium hexafluorophosphate (HATU) (0.22 g, $0.59 \mathrm{mmol})$ at room temrature. The reaction mixture was heated at $50{ }^{\circ} \mathrm{C}$ for $15-45$ min. The mixture was dissolved in ethyl acetate, washed sequentially with water and saturated sodium bicarbonate solution, dried over sodium sulfate and concentrated in vacuo. The crude product was purified by column chromatography $\left(\mathrm{SiO}_{2}, 10-30 \%\right.$ ethyl acetate $/ n$-hexane).

N-((13aS)-9-Oxo-11,12,13,13a-tetrahydro-9H-benzo[e]pyrrolo[1,2-a][1,2,4]triazolo[3,4-c][1,4]diaze pin-3-yl)benzamide (7b). Yield 80\%; off-white solid; mp $173-175^{\circ} \mathrm{C}$; IR $v_{\max } 3442,1641,1582,1332$, $699 \mathrm{~cm}^{-1}$; ${ }^{1} \mathrm{H}-\mathrm{NMR}: \delta 2.0-2.05(2 \mathrm{H}, \mathrm{m}), 2.31-2.40(1 \mathrm{H}, \mathrm{m}), 2.80-2.85(1 \mathrm{H}, \mathrm{m}), 3.50-3.74(2 \mathrm{H}, \mathrm{m})$, 
4.82-4.84 (1H, m), 7.40-7.62 (6H, m), 7.82-8.00 (3H, m), $11.22(1 \mathrm{H}, \mathrm{br} \mathrm{s}, \mathrm{NH}) ; \mathrm{LCMS}\left(\mathrm{ES}^{+}\right): m / z$ $360[\mathrm{M}+\mathrm{H}]^{+}$.

2-Fluoro-N-((13aS)-9-oxo-11,12,13,13a-tetrahydro-9H-benzo[e]pyrrolo[1,2-a][1,2,4]triazolo[3,4-c][1,4] diazepin-3-yl)benzamide (7c). Yield 75\%; white solid; mp 180-181 ${ }^{\circ} \mathrm{C}$; IR $v_{\max } 3437,1647,1572$, 1326, $827 \mathrm{~cm}^{-1}$; ${ }^{1} \mathrm{H}-\mathrm{NMR}: \delta 1.98-2.03$ (2H, m), 2.31-2.40 (1H, m), 2.81-2.89 (1H, m), 3.51-3.72 (2H, $\mathrm{m}), 4.82-4.84(1 \mathrm{H}, \mathrm{m}), 7.23-7.38(1 \mathrm{H}, \mathrm{m}), 7.53-7.72(5 \mathrm{H}, \mathrm{m}), 7.86-7.94(2 \mathrm{H}, \mathrm{m}), 11.20(1 \mathrm{H}$, br s, $\mathrm{NH}) ; \mathrm{LCMS}\left(\mathrm{ES}^{+}\right): m / z 378[\mathrm{M}+\mathrm{H}]^{+}$.

3-Fluoro-N-((13aS)-9-oxo-11,12,13,13a-tetrahydro-9H-benzo[e]pyrrolo[1,2-a][1,2,4]triazolo[3,4-c][1,4] diazepin-3-yl)benzamide (7d). Yield 80\%; white solid; mp 159-160 ${ }^{\circ} \mathrm{C}$; IR $v_{\max } 3437,1640,1579$, 1342, $765 \mathrm{~cm}^{-1}$; ${ }^{1} \mathrm{H}-\mathrm{NMR}: \delta$ 1.98-2.04 $(2 \mathrm{H}, \mathrm{m}), 2.29-2.41(1 \mathrm{H}, \mathrm{m}), 2.78-2.82(1 \mathrm{H}, \mathrm{m}), 3.52-3.71$ $(2 \mathrm{H}, \mathrm{m}), 4.82-4.84(1 \mathrm{H}, \mathrm{m}), 7.36-7.42(1 \mathrm{H}, \mathrm{m}), 7.48-7.56(2 \mathrm{H}, \mathrm{m}), 7.63-7.92(5 \mathrm{H}, \mathrm{m})$; LCMS $\left(\mathrm{ES}^{+}\right)$: $m / z 378[\mathrm{M}+\mathrm{H}]^{+}$.

4-Fluoro-N-((13aS)-9-oxo-11,12,13,13a-tetrahydro-9H-benzo[e]pyrrolo[1,2-a][1,2,4]triazolo[3,4-c][1,4] diazepin-3-yl)benzamide (7e). Yield 75\%; white solid; mp 319-321 ${ }^{\circ} \mathrm{C}$; IR $v_{\max } 3435,1608,1559,1338$, $778 \mathrm{~cm}^{-1}$; ${ }^{1} \mathrm{H}-\mathrm{NMR}: \delta$ 2.00-2.05 (2H, m), 2.31-2.40 (1H, m), 2.82-2.85 (1H, m), 3.50-3.78 (2H, m), 4.82-4.84 (1H, m), 7.20-7.25 (1H, m), 7.38-7.42 (1H, m), 7.55-7.63 (2H, m), 7.92-7.96 (2H, m), 8.12-8.19 (2H, m), $11.23\left(1 \mathrm{H}\right.$, br s, NH); LCMS $\left(\mathrm{ES}^{+}\right): m / z 378[\mathrm{M}+\mathrm{H}]^{+}$.

3-Methoxy-N-((13aS)-9-oxo-11,12,13,13a-tetrahydro-9H-benzo[e]pyrrolo[1,2-a][1,2,4]triazolo[3,4-c] [1,4]diazepin-3-yl)benzamide (7f). Yield 70\%; off-white solid; mp 168-170 ${ }^{\circ} \mathrm{C}$; IR $v_{\max } 3454,1639$, 1590, 1327, $685 \mathrm{~cm}^{-1}$; ${ }^{1} \mathrm{H}-\mathrm{NMR}: \delta 2.00-2.05(2 \mathrm{H}, \mathrm{m}), 2.35-2.41(1 \mathrm{H}, \mathrm{m}), 2.80-2.85(1 \mathrm{H}, \mathrm{m})$, 3.55-3.61 (1H, m), 3.62-3.69 (1H, m), 3.80 (3H, s), 4.82-4.84 (1H, m), 7.15-7.21 (1H, m), 7.39-7.64 $(6 \mathrm{H}, \mathrm{m}), 7.89-7.93(1 \mathrm{H}, \mathrm{m}), 11.21(1 \mathrm{H}, \mathrm{br} \mathrm{s}, \mathrm{NH})$; LCMS $\left(\mathrm{ES}^{+}\right): m / z 390[\mathrm{M}+\mathrm{H}]^{+}$.

3-Cyano-N-((13aS)-9-oxo-11,12,13,13a-tetrahydro-9H-benzo[e]pyrrolo[1,2-a][1,2,4]triazolo[3,4-c] [1,4]diazepin-3-yl)benzamide (7g). Yield 65\%; pale yellow solid; mp $245-246{ }^{\circ} \mathrm{C}$; IR $v_{\max } 3435,2217$, 1645, 1535, $651 \mathrm{~cm}^{-1}$; ${ }^{1} \mathrm{H}-\mathrm{NMR}: \delta 1.98-2.03(2 \mathrm{H}, \mathrm{m}), 2.31-2.42(1 \mathrm{H}, \mathrm{m}), 2.76-2.81(1 \mathrm{H}, \mathrm{m})$, 3.51-3.71 (2H, m), 4.82-4.84 (1H, m), 7.54-7.76 (3H, m), 7.91-8.02 (3H, m), 8.24-8.37 (2H, m); LCMS $\left(\mathrm{ES}^{+}\right): m / z 385[\mathrm{M}+\mathrm{H}]^{+}$.

3-Chloro-N-((13aS)-9-oxo-11,12,13,13a-tetrahydro-9H-benzo[e]pyrrolo[1,2-a][1,2,4] triazolo[3,4-c][1,4] diazepin-3-yl)benzamide (7h). Yield 65\%; off-white solid; mp $175-177{ }^{\circ} \mathrm{C}$; IR $v_{\max } 3434,1642,1565$, 1335, $788 \mathrm{~cm}^{-1}$; ${ }^{1} \mathrm{H}-\mathrm{NMR}: \delta$ 1.98-2.03 $(2 \mathrm{H}, \mathrm{m}), 2.31-2.40(1 \mathrm{H}, \mathrm{m}), 2.76-2.81(1 \mathrm{H}, \mathrm{m}), 3.51-3.75$ $(2 \mathrm{H}, \mathrm{m}), 4.82-4.84(1 \mathrm{H}, \mathrm{m}), 7.48-7.78(4 \mathrm{H}, \mathrm{m}), 7.88-7.98(4 \mathrm{H}, \mathrm{m})$; LCMS $\left(\mathrm{ES}^{+}\right): \mathrm{m} / z 394[\mathrm{M}+\mathrm{H}]^{+}$.

$N$-((13aS)-9-Oxo-11,12,13,13a-tetrahydro-9H-benzo[e]pyrrolo[1,2-a][1,2,4]triazolo[3,4-c][1,4]diaze pin-3-yl)-3-(trifluoromethyl)benzamide (7i). Yield 70\%; white solid; mp 234-236 ${ }^{\circ} \mathrm{C}$; IR $v_{\max } 3431$, 1645, 1580, $756 \mathrm{~cm}^{-1}$; ${ }^{1} \mathrm{H}-\mathrm{NMR}: \delta 2.00-2.05(2 \mathrm{H}, \mathrm{m}), 2.35-2.41(1 \mathrm{H}, \mathrm{m}), 2.79-2.82(1 \mathrm{H}, \mathrm{m})$, 3.52-3.60 (1H, m), 3.67-3.75 (1H, m), 4.82-4.84 (1H, m), 7.57-7.60 (1H, m), 7.65-7.75 (2H, m), 7.92-7.98 (3H, m), 8.22-8.35 (2H, m); LCMS $\left(\mathrm{ES}^{+}\right): m / z 428[\mathrm{M}+\mathrm{H}]^{+}$. 
N-((13aS)-9-Oxo-11,12,13,13a-tetrahydro-9H-benzo[e]pyrrolo[1,2-a][1,2,4]triazolo[3,4-c][1,4]diazepin3-yl)nicotinamide (7j). Yield 75\%; pale yellow solid; mp $225{ }^{\circ} \mathrm{C}$; IR $v_{\max } 3445,2923,2360,1700$, 1628, 1229, $759 \mathrm{~cm}^{-1}$; ${ }^{1} \mathrm{H}-\mathrm{NMR}: \delta 2.00-2.05(2 \mathrm{H}, \mathrm{m}), 2.37-2.41(1 \mathrm{H}, \mathrm{m}), 2.78-2.82(1 \mathrm{H}, \mathrm{m})$, 3.52-3.68 (2H, m), 4.82-4.85 (1H, m), 7.42-7.61 (2H, m), 7.91-7.95 $(1 \mathrm{H}, \mathrm{m}), 8.21-8.35(2 \mathrm{H}, \mathrm{m})$, 8.67-8.80 (1H, m), 7.92-7.96 (1H, m), 9.12-9.20 (1H, m), $11.55(1 \mathrm{H}, \mathrm{br} \mathrm{s}, \mathrm{NH})$; LCMS $\left(\mathrm{ES}^{+}\right): m / z$ $361[\mathrm{M}+\mathrm{H}]^{+}$.

N-((13aS)-9-Oxo-11,12,13,13a-tetrahydro-9H-benzo[e]pyrrolo[1,2-a][1,2,4]triazolo[3,4-c][1,4]diazepin3-yl)thiophene-2-carboxamide (7k). Yield 72\%; off-white solid; mp $185-186{ }^{\circ} \mathrm{C}$; IR $v_{\max } 3425,1635$, $1561 \mathrm{~cm}^{-1}$; ${ }^{1} \mathrm{H}-\mathrm{NMR}: \delta 2.00-2.05(2 \mathrm{H}, \mathrm{m}), 2.31-2.40(1 \mathrm{H}, \mathrm{m}), 2.85-2.92(1 \mathrm{H}, \mathrm{m}), 3.45-3.65(2 \mathrm{H}, \mathrm{m})$, 4.81-4.83 (1H, m), 7.02-7.22 (1H, m), 7.42-7.62 (3H, m), 7.90-8.20 (3H, m), $11.28(1 \mathrm{H}$, br s, NH); LCMS $\left(\mathrm{ES}^{+}\right): m / z 366[\mathrm{M}+\mathrm{H}]^{+}$.

\subsection{Biological Activity Test Procedures}

\subsubsection{Cell Culture}

Human fibroblast cells (MRC-5) cells were used for cell culture experiments. Cells were cultured with 90\% Eagle's minimum essential medium with $2 \mathrm{mM}$ L-glutamine and Earle's BSS adjusted to contain $1.5 \mathrm{~g} / \mathrm{L}$ sodium bicarbonate, $0.1 \mathrm{mM}$ non-essential amino acids, $1.0 \mathrm{mM}$ sodium pyruvate with $10 \%$ fetal bovine serum, $100 \mathrm{U} / \mathrm{mL}$ penicillin, and $100 \mu \mathrm{g} / \mathrm{mL}$ streptomycin. The cells were placed in an incubator to maintain high humidity, $37{ }^{\circ} \mathrm{C}$, and $5 \% \mathrm{CO}_{2}$ for the cell culture experiments. Cells were routinely sub-passaged in a 1:2 to $1: 5$ split using $0.25 \%$ trypsin. Human hepatocellular carcinoma (Mahlavu) epithelial cells were used for cell culture experiments. Cells were cultured with $90 \%$ Dulbecco's modified Eagle's medium with $10 \%$ fetal bovine serum, $100 \mathrm{U} / \mathrm{mL}$ penicillin, and $100 \mu \mathrm{g} / \mathrm{mL}$ streptomycin. The cells were placed in an incubator to maintain high humidity, a temperature of $37^{\circ} \mathrm{C}$, and $5 \% \mathrm{CO}_{2}$ for cell culture experiments. Cells were routinely sub-passaged in a 1:4 to 1:6 split using $0.25 \%$ trypsin.

\subsubsection{CCK-8 for Cell Proliferation Assay}

The cell viability was determined using a CCK-8 assay following the manufacturer's instructions. Mahlavu cells and MRC-5 cells were seeded in 96-well plates and allowed to attach overnight at $37^{\circ} \mathrm{C}$. Cells were treated with PBDT-7a-k in 0.1\% FBS MEM or DMEM (100 $\mu \mathrm{L} /$ well), and the cells were incubated for $48 \mathrm{~h}$ in a humidified atmosphere. After the incubation period, $10 \mu \mathrm{L}$ of cell Counting Kit- 8 solution was added to the medium, and the cells were incubated for an additional $1-4 \mathrm{~h}$ in the incubator. The absorbance at $450 \mathrm{~nm}$ was then measured using a microplate reader. The IR was calculated using the following equation: $I R=[1-$ (A value for the treated samples - A value for the blank samples)/(A value for the control samples - A value for the blank samples) $] \times 100 \%$. The assays were performed in triplicate and repeated at least twice. 


\section{Conclusions}

We have synthesized a series of novel amido-substituted triazolopyrrolo [2,1-c][1,4]benzodiazepines from isatoic anhydride in high total yields. The biological evaluation of these compounds based on the growth of Mahlavu and MRC-5 cells revealed that compound PBDT-7i selectively inhibited the proliferation of Mahlavu cells. More interestingly, compound PBDT-7i might inhibit not only Mahlavu cell growth, but also shows low toxicity towards MRC-5 cells.

\section{Acknowledgements}

This work was supported by the GVK Biosciences and National Science Council, Taiwan (NSC 100-2320-B-182A-006-)(NSC 101-2113-M-039 -004 -MY2). The authors are thankful to Srinivasa Rao Alapati and Balaram Patro for their valuable suggestions and support.

\section{References}

1. McGlynn, K.A.; Tsao, L.; Hsing, A.W.; Devesa, S.S.; Fraumeni, J.F., Jr. International trends and patterns of primary liver cancer. Int. J. Cancer 2001, 94, 290-296.

2. Li, L.Y.; Dai, H.Y.; Yeh, F.L.; Kan, S.F.; Lang, J.; Hsu, J.L.; Jeng, L.B.; Chen, Y.H.; Sher, Y.P.; Lin, W.C.; Hung, M.C. Targeted hepatocellular carcinoma proapoptotic BikDD gene therapy. Oncogene 2011, 30, 1773-1783.

3. McCall, W.V. A psychiatric perspective on insomnia. J. Clin. Psychitr. 2001, 62, 27-32.

4. Hsu, M.C.; Schutt, A.D.; Holly, M.; Slice, L.W.; Sherman, M.I.; Richman, D.D.; Potash, M.J.; Volsky, D.J. Inhibition of HIV replication in acute and chronic infections in vitro by a Tat antagonist. Science 1991, 254, 1799-1802.

5. Micale, N.; Vairagoundar, R.; Yakovlev, A.G.; Kozikowski, A.P. Design and synthesis of a potent and selective peptidomimetic inhibitor of caspase-3. J. Med. Chem. 2004, 47, 6455-6458.

6. De Corte, B.L. From 4,5,6,7-Tetrahydro-5-methylimidazo[4,5,1-jk](1,4) benzodiazepin -2(1H) -one (TIBO) to Etravirine (TMC125): Fifteen years of research on non-nucleoside inhibitors of HIV-1 reverse transcriptase. J. Med. Chem. 2005, 48, 1689-1696.

7. Hadac, E.M.; Dawson, E.S.; Darrow, J.W.; Sugg, E.E.; Lybrand, T.P.; Miller, L.J. Novel benzodiazepine photoaffinity probe stereoselectively labels a site deep within the membrane-spanning domain of the cholecystokinin receptor. J. Med. Chem. 2006, 49, 850-863.

8. Primofiore, G.; Da Settimo, F.; Taliani, S.; Salerno, S.; Novellino, E.; Greco, G.; Cosimelli, B.; Besnard, F.; Costa, B.; Montali, M.; Martini, C. High affinity central benzodiazepine receptor ligands: Synthesis and biological evaluation of a series of phenyltriazolobenzotriazindione derivatives. J. Med. Chem. 2005, 48, 2936-2943.

9. Thurston, D.E. Advances in the study of pyrrolo [2,1-c][1,4]-benzodiazepine (PBD) antitumour antibiotics. In Molecular Aspects of Anticancer Drug-DNA Interactions; Neidle, S., Waring, M.J., Eds.; The Macmillan Press Ltd.: London, UK, 1993; pp. 54-88.

10. Thurston, D.E.; Bose, D.S. Synthesis of DNA-interactive pyrrolo[2,1-c][1,4]benzodiazepines. Chem. Rev. 1994, 94, 433-465. 
11. Thomas, A.W. A concise route to triazolobenzodiazepine derivatives via a one-pot alkyne-azide cycloaddition reaction. Bioorg. Med. Chem. Lett. 2002, 12, 1881-1884.

12. Roger-Evans, M.; Spurr, P.; Hennig, M. The isolation and use of a benzodiazepine iminochloride for the efficient construction of flumazenil. Tetrahedron Lett. 2003, 44, 2425-2428.

13. Broggini, G.; Molteni, G.; Terraneo, A.; Zecchi, G. A facile synthesis of flumazenil analogues. Tetrahedron 1999, 55, 14803-14806.

14. Gu, Z.-Q.; Wong, G.; Dominguez, C.; de Costa, B.R.; Rice, K.C.; Skolnick, P. Synthesis and evaluation of imidazo [1,5-a][1,4] benzodiazepine esters with high affinities and selectivities at diazepam-insensitive benzodiazepine receptors. J. Med. Chem. 1993, 36, 1001-1006.

15. Brabcova, R.; Kubova, H.; Velisek, L.; Mares, P. Effects of a benzodiazepine, bretazenil (Ro 16-6028), on rhythmic metrazol EEG activity: Comparison with standard anticonvulsants. Epilepsia 1993, 34, 1135-1140.

16. Naito, Y.; Akahoshi, F.; Takeda, S.; Okada, T.; Kajii, M.; Nishimura, H.; Sigiura, M.; Fukaya, C.; Kagitani, Y. Synthesis and pharmacological activity of triazole derivatives inhibiting eosinophilia. J. Med. Chem. 1996, 39, 3019-3029.

17. Cho, J.H.; Bernard, D.L.; Sidwell, R.W.; Kern, E.R.; Chu, C.K. Synthesis of cyclopentenyl carbocyclic nucleosides as potential antiviral agents against orthopoxviruses and SARS. J. Med. Chem. 2006, 49, 1140-1148.

18. Vijaya Raj, K.K.; Narayana, B.; Suchetha Kumari, N.; Ashalatha, B.V.; Sarojini, B.K. Neuropsychobehavioral effects and anticancer activity of some substituted triazolo[4,3-a][1,4]benzodiazepines. J. Pharmacol. Toxicol. 2006, 1, 471-477.

19. Kumaraswamy, S.; Mukkanti, K.; Srinivas, P. Palladium catalyzed synthesis of quinazolino $[1,4]$ benzodiazepine alkaloids and analogous. Tetrahedron 2012, 68, 2001-2006.

20. Foloppe, M.-P.; Rault, I.; Rault, S.; Robba. M. Pyrrolo[2,1-c][1,4]benzodiazepines: Synthesis of $\mathrm{N}$-Substituted Amidines. Heterocycles 1993, 36, 63-69.

21. Hester, J.B., Jr.; Chidester, C.G.; Szmuszkovicz, J. Synthesis and chemistry of $N$-methyl-6-phenyl4H-s-triazolo[4,3-a][1,4]benzodiazepinium derivatives. J. Org. Chem. 1974, 44, 2688-2693.

Sample Availability: Samples of compounds PBDT-7a-k are available from the authors.

(C) 2012 by the authors; licensee MDPI, Basel, Switzerland. This article is an open access article distributed under the terms and conditions of the Creative Commons Attribution license (http://creativecommons.org/licenses/by/3.0/). 